\title{
Obscured active galactic nuclei triggered in compact star-forming galaxies
}

\author{
Yu-Yen Chang, ${ }^{1 \star}$ Emeric Le Floc'h, ${ }^{1}$ Stéphanie Juneau, ${ }^{1}$ Elisabete da Cunha, ${ }^{2}$ \\ Mara Salvato, ${ }^{3}$ Francesca Civano, ${ }^{4,5}$ Stefano Marchesi, ${ }^{4,5,6}$ J. M. Gabor, ${ }^{1}$ \\ Olivier Ilbert, ${ }^{7}$ Clotilde Laigle, ${ }^{8}$ H. J. McCracken, ${ }^{8}$ Bau-Ching Hsieh ${ }^{9}$ \\ and Peter Capak ${ }^{10,11}$ \\ ${ }^{1}$ CEA Saclay, DSM/Irfu/Service d'Astrophysique, Orme des Merisiers, F-91191 Gif-sur-Yvette Cedex, France \\ ${ }^{2}$ Mt Stromlo Observatory, The Australian National University, Cotter Rd, Weston Creek, ACT 2611, Australia \\ ${ }^{3}$ Max-Planck-Institut für Plasma Physik and Excellence Cluster, D-85748 Garching, Germany \\ ${ }^{4}$ Harvard-Smithsonian Center for Astrophysics, 60 Garden Street, Cambridge, MA 02138, USA \\ ${ }^{5}$ Yale Center for Astronomy and Astrophysics, 260 Whitney Avenue, New Haven, CT 06520, USA \\ ${ }^{6}$ Dipartimento di Fisica e Astronomia, Universit'a di Bologna, viale Berti Pichat 6/2, I-40127 Bologna, Italy \\ ${ }^{7}$ LAM (Laboratoire d'Astrophysique de Marseille), Aix-Marseille Université, CNRS, UMR 7326, F-13388 Marseille, France \\ ${ }^{8}$ Sorbonne Université, UPMC University Paris 06, et CNRS, UNR 7095, IAP, 98 b bd Arago, F-75014 Paris, France \\ ${ }^{9}$ ASIAA Sinica, AS/NTU. No. 1, Section 4, Roosevelt Rd., Taipei 10617, Taiwan, R.O.C. \\ ${ }^{10}$ Caltech, 1200 E. California Blvd., Pasadena, CA 91125, USA \\ ${ }^{11}$ IPAC, 1200 E. California Blvd., Pasadena, CA 91125, USA
}

Accepted 2016 December 6. Received 2016 December 6; in original form 2016 July 12

\begin{abstract}
We present a structural study of 182 obscured active galactic nuclei (AGNs) at $z \leq 1.5$, selected in the Cosmic Evolution Survey field from their extreme infrared to X-ray luminosity ratio and their negligible emission at optical wavelengths. We fit optical to far-infrared spectral energy distributions and analyse deep Hubble Space Telescope imaging to derive the physical and morphological properties of their host galaxies. We find that such galaxies are more compact than normal star-forming sources at similar redshift and stellar mass, and we show that it is not an observational bias related to the emission of the AGN. Based on the distribution of their $U V J$ colours, we also argue that this increased compactness is not due to the additional contribution of a passive bulge. We thus postulate that a vast majority of obscured AGNs reside in galaxies undergoing dynamical compaction, similar to processes recently invoked to explain the formation of compact star-forming sources at high redshift.
\end{abstract}

Key words: galaxies: active-galaxies: star formation-infrared: galaxies.

\section{INTRODUCTION}

The census of the population of X-ray and optically selected active galactic nuclei (AGNs) has undergone dramatic improvements over the past few years, and a general consensus on the nature of their host galaxies has now emerged : The vast majority of AGNs reside in normal isolated galaxies, in which the activity of star formation co-evolves with the super-massive black hole accretion (Cisternas et al. 2011; Kocevski et al. 2012; Mullaney et al. 2012; Juneau et al. 2013). However, an ongoing debate still persists on the exact structural properties of the AGN host galaxies. When compared to normal star-forming discs at $z \sim 1$, X-ray AGN hosts have been found either bulgier (Grogin et al. 2005; Pierce et al. 2007), similar (Böhm et al. 2013; Villforth et al. 2014) or intermediate between discs and spheroids (Gabor et al. 2009). Such inconsistent results might be partly explained by the difficulty to separate the contribution of the central AGN from the emission of the underlying host, even when high resolution images such as those obtained with the Hubble Space Telescope (HST) are used.

Obscured AGNs weakly contribute to the total galaxy emission at optical wavelengths, and the characterization of their host is therefore less subject to the subtraction of the AGN signature. Interestingly, analysis of X-ray-selected Compton-thick AGNs and their host galaxies has revealed a fraction of disturbed morphologies and mergers increasing with obscuration, substantially larger than found among moderately obscured and unobscured AGNs (Kocevski et al. 2015; Lanzuisi et al. 2015). Yet, most of the obscured AGNs studied so far have among the highest bolometric luminosities measured $\left(L_{\text {bol }}>10^{43} \mathrm{~L} \odot\right)$ and may not be representative of the bulk of the obscured AGN population.

In this Letter, we identify AGNs from their characteristic mid-IR power-law emission using the Cosmic Evolution Survey (COSMOS, Scoville et al. 2007) and focus on a sub-sample of 
obscured AGNs carefully selected based on their infrared-to-Xray luminosity ratio. Supplementing the more traditional X-ray Compton-thick selection, our technique provides us with a large and complementary sample of obscured AGNs at moderate luminosities, allowing a more complete view on the properties of the galaxies hosting this population. We use AB magnitudes and adopt the cosmological parameters $\left(\Omega_{\mathrm{M}}, \Omega_{\Lambda}, h\right)=(0.35,0.70,0.70)$ and the Chabrier (2003) stellar initial mass function.

\section{THE DATA}

\subsection{Parent galaxy sample and IR-AGN candidates}

Infrared-luminous AGN (IR-AGN) candidates were identified among the $24 \mu \mathrm{m}$ sources of the COSMOS field (Le Floc'h et al. 2009) using a criterion similar to that initially proposed by Lacy et al. (2004). In practice, we first considered all sources with IRAC $F_{v}$ flux densities monotonically rising from 3.6 to $8 \mu \mathrm{m}$, as well as those with IRAC 5.8 and $8 \mu \mathrm{m}$ fluxes exceeding by $>3 \sigma$ the expected emission of the stellar component assuming typical galaxy models and spectral energy distribution (SED) fitting techniques. In the parameter space defined by the $F_{5.8 \mu \mathrm{m}} / F_{3.6 \mu \mathrm{m}}$ and $F_{8 \mu \mathrm{m}} / F_{4.5 \mu \mathrm{m}}$ colours, these objects populate a specific area that shows also minimized contamination by star-forming galaxies at low to intermediate redshifts (Chang et al., in preparation). Assuming the latest photometric and spectroscopic redshifts in COSMOS, all $24 \mu \mathrm{m}$ sources at $z \leq 1.5$ located in this two-colour region were hence selected as potential AGN-dominated galaxies. This led to a total sample of 655 IR-AGN candidates, 353 of which are also detected in the deep X-ray Chandra COSMOS Legacy with an estimated $L_{2-10 \mathrm{keV}}$ luminosity greater than $10^{42} \mathrm{erg} \mathrm{s}^{-1}$ (Civano et al. 2016; Marchesi et al. 2016). Among the 655 selected AGNs, 426 have reliable spectroscopy. For the other 229 sources, 106 are X-ray detected, and we take the photometric redshifts from Marchesi et al. (2016) as described in Salvato et al. (2011). For the remaining 123 AGNs, we assumed that the optical/near-infrared (NIR) emission is mostly powered by the host galaxies and thus used the photometric redshifts derived by Laigle et al. (2016).

All COSMOS $24 \mu \mathrm{m}$ sources have optical to far-IR photometry available from the band-merged COSMOS2015 catalogue of Laigle et al. (2016). We fit SEDs to the IR-AGNs with a new version of MAGPHYS (da Cunha, Charlot \& Elbaz 2008; da Cunha et al. 2015, in preparation), updated to account for a possible AGN contribution and relying on both obscured and unobscured AGN templates. Fig. 1 shows the multiband photometry of one IR-AGN candidate and its host galaxy, along with the best SED fit decomposed into the relative AGN and stellar contributions. We find that galaxies hosting IR-AGNs at $z \leq 1.5$ in COSMOS have typical stellar masses $M_{*} \gtrsim 10^{10} \mathrm{M}_{\odot}$ with a peak distribution at $M_{*} \sim 10^{10.5} \mathrm{M}_{\odot}$. They have specific star formation rates (sSFR) typical of star-forming sources, 45 per cent of them being individually detected with Herschel in the far-IR, i.e. in a wavelength range where the dust emission is mostly powered by intense star formation.

\subsection{Obscured AGNs}

Assuming the total IR luminosity of the AGN component derived from the SED fits $\left(L_{\mathrm{IR}, \mathrm{AGN}}\right)$ we define 'obscured AGNs' as IR-AGNs with $L_{\mathrm{IR}, \mathrm{AGN}} / L_{\mathrm{X}}>20$, where $L_{\mathrm{X}}$ is the X-ray $2-10 \mathrm{keV}$ luminosity or upper limit obtained from the Chandra Legacy observations of COSMOS. For the latter, we assumed $\Gamma=1.4$ following the prescriptions of Marchesi et al. (2016). We used the 90 per cent

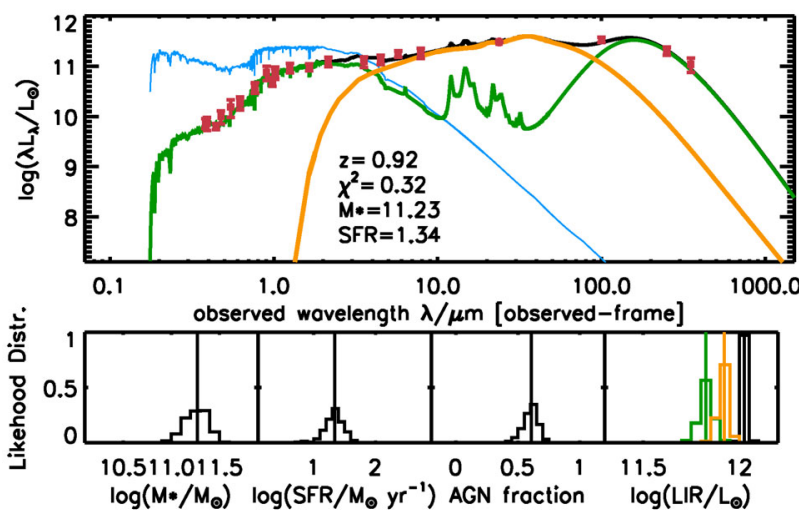

Figure 1. The multiband photometry of a type-2 IR-AGN (top panel, red data points) along with its best SED fit (black solid line). The emission from the stellar component and the AGN are represented by the green and orange curves, respectively, while the blue line shows the unattenuated stellar spectrum. Likelihood distributions are illustrated for the stellar mass, the SFR, AGN fraction (contribution to the IR) and IR luminosity.

completeness flux limit $f_{2}-10 \mathrm{kev}=5.0 \times 10^{-15} \mathrm{erg} \mathrm{s}^{-1} \mathrm{~cm}^{-2}$ in the case of no X-ray detection, resulting in a selection of 265 objects. Given the relation $L_{\mathrm{IR}} \sim 2 \times L_{6 \mu \mathrm{m}}$ implied by our Type- 2 AGN SED models, we note that our criterion identifies AGNs with dust reprocessed emission at least 0.3 dex larger than expected from the intrinsic $L_{6 \mu \mathrm{m}}-L_{\mathrm{X}}$ relationship observed among unobscured AGNs (e.g. Stern 2015). Therefore, our selection is not restrictive to extreme levels of X-ray absorption [e.g. a Compton-thick AGN with $N_{\mathrm{H}}=10^{24} \mathrm{~cm}^{-2}$ would correspond to $L_{\mathrm{IR}} / L_{\mathrm{X}} \sim 80$, Fiore et al. (2009)] but it provides a statistically large sample of AGNs with high levels of obscuration, among which $\sim 50$ per cent escape the standard X-ray selection criterion $L_{\mathrm{X}}>10^{42} \mathrm{erg} \mathrm{s}^{-1}$. Because of small-scale dust-free gas absorbers within the broad line region, AGNs with strong obscuration in the X-rays can still contribute some light at UV/optical wavelengths (e.g. Lanzuisi et al. 2013; Merloni et al. 2014). To avoid skewing our morphology measurements, 34 sources with detectable AGN contribution in the optical were excluded from our sample: Based on our SED fitting, we kept only sources where the AGN is best described by a deeply obscured torus emission contributing less than 0.001 per cent to the $I$ band, ensuring that the optical light is fully dominated by the stellar component of the host galaxy, as shown in Fig. $1 .{ }^{1}$ Independently of our SED fits, we also removed 17 AGNs previously identified as type 1 by Salvato et al. (2011) and Lilly et al. (2009). Restricting our analysis to the $1.64 \mathrm{deg}^{2} \mathrm{HST} / \mathrm{ACS}$ imaging of COSMOS, we were left with a final sample of 182 obscured AGNs at $z \leq 1.5$.

\section{MORPHOLOGY}

We analysed the morphology of the obscured AGN host galaxies using the I-band ACS imaging of COSMOS, with both a visual classification approach and a parametric two-dimensional surface brightness fitting method. While the visually classified properties will be reported in a future publication, we discuss hereafter the results obtained by fitting the $I$-band light distribution with the GALFIT algorithm (Peng et al. 2010). Having eliminated all the sources with

\footnotetext{
${ }^{1}$ We made the stacking of the probability distribution functions of the AGN component resulting from the decomposition of each source in our sample and confirm a very high probability ( $>96.95$ per cent) to host a deeply buried AGN with no detectable emission $(<0.001$ per cent) in the $I$ band.
} 

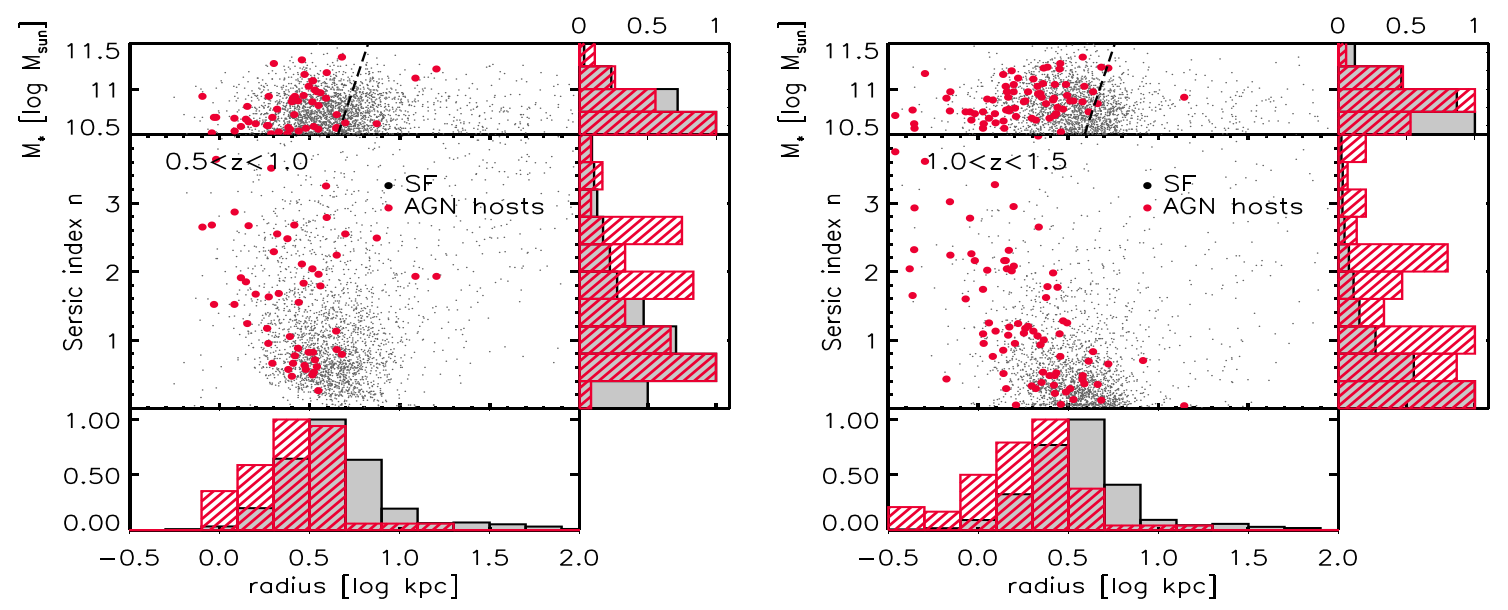

Figure 2. Stellar mass and Sérsic index versus half-light radius of obscured AGN hosts (red dots), compared to star-forming galaxies (black) at $0.5<z<1$ (left-hand panel) and $1<z<1.5$ (right-hand panel). The corresponding distributions are represented with normalized histograms along the $x$ - and $y$-axis. The hosts of obscured AGNs have smaller radii and higher Sérsic indices than star-forming sources. The dashed-line indicates the mass-size relation of star-forming galaxies reported by van der Wel et al. (2014).

detectable AGN contribution and avoiding degeneracy from multiple components, it is fair to assume that the HST image can be fitted by using a Sérsic profile only as it is confirmed by radial light profile later. Fig. 2 shows the distributions of their half-light radius and Sérsic index measured for $M_{*}>10^{10.5} \mathrm{M}_{\odot}$ at $0.5<z<1$ and $1<z<1.5$, compared to the distributions obtained for a control sample of star-forming galaxies selected from the COSMOS2015 catalogue (Laigle et al. 2016) at similar redshifts and comparable stellar masses.

It is clear that the host galaxies of obscured AGNs have on average smaller radii and larger Sérsic indices, a result confirmed at $>99$ per cent confidence by a Kolmogorov-Smirnov (K-S) test. These galaxies could thus share some properties with the hosts of X-ray bright and radio-selected AGNs, which also seem to be more bulgy than star-forming galaxies even after subtracting the emission from the AGN (Gabor et al. 2009; Bruce et al. 2016). Similarly, they appear to be shifted towards smaller sizes with respect to the mass-size relation of star-forming galaxies (van der Wel et al. 2014).

To further quantify this trend, we investigated the averaged galaxy radial light profile using a stacking analysis. For each object, an ACS image centred on the peak emission of the best-fitting twodimensional model given by GALFIT was extracted and re-sampled to a common physical scale of $0.18 \mathrm{kpc}$ pixel $^{-1}$ using bilinear interpolation. The sample of star-forming galaxies was matched in stellar mass to the AGNs, and an averaged stack was created separately for the two populations in the two redshift bins mentioned above. Fig. 3 shows the corresponding stacked images along with the average radial profile of the associated stacked detection, as well as the structural parameters of the best fits obtained with GALFIT. Here again, the I-band light distribution characterizing the hosts of obscured AGNs appears more compact than the profile of starforming galaxies. If we further sub-divide and compare the two populations by bin of galaxy stellar mass, the same trend is still observed for each redshift range, albeit with a lower significance at $M_{*} \gtrsim 10^{11} \mathrm{M}_{\odot}$ probably altered by the lower signal-to-noise ratio at the most massive end due to small sample size.

We stress that this steeper light profile of AGN hosts cannot be due to the impact of the central AGN nor due to the presence of a compact circum-nuclear starburst. Considering that in such cases the emission would be unresolved at the resolution of HST, we used GALFIT to analyse the AGN stacked images assuming the combination of a Sérsic disc and a PSF. Fixing the geometry of the first component to that obtained for the control sample of starforming sources and adopting a PSF does not lead to acceptable fits. This profile overpredicts the central flux while decreasing too steep on small scales $(<1 \mathrm{kpc})$. Similarly, leaving the Sérsic index and the radius as free parameters results in a small PSF fraction ( $\lesssim 3.5$ per cent) and thus a disc component again much steeper than the average profile of star-forming galaxies (see Fig. 3). The more compact profile we observe among obscured AGNs is therefore intrinsically related to the morphology of their hosts, suggesting either a global compaction of the star-forming disc or the presence of an additional compact core in the central region.

We tested the latter option with the hypothesis that an old stellar bulge has already formed in these objects. We considered the COSMOS red passive sources selected at similar redshift and stellar mass using the $n U V r J$ colour-colour criterion from Laigle et al. (2016) and derived their average radial light profile, as described above. As illustrated in Fig. 3, we find that the total light profile of obscured AGN hosts cannot be reliably reproduced by combining a red stellar bulge with the typical disc component characterizing the star-forming galaxies of the field (colour-coded swath). Furthermore, the stack modelling suggests that the contribution of a possible red bulge to the total I-band light should be larger than $\sim 60$ per cent in order to steepen the radial profile as observed, which is in strong disagreement with the colour distribution of AGN host galaxies at optical wavelengths. For instance, we show in Fig. 4 the standard $U V J$ rest-frame colour plot obtained for the hosts of obscured AGNs (assuming the best-fitting stellar component derived from our SED fitting), compared to the control sample of star-forming galaxies. Assuming a typical passive galaxy SED, an $\sim 60$ per cent bulge contribution to the $I$-band light would result in $U-V$ colours statistically redder for the AGN hosts, while the two samples exhibit remarkably similar colour distributions. We thus conclude that the apparent compactness of obscured AGN host galaxies is not produced by the additional contribution of an old stellar bulge. 


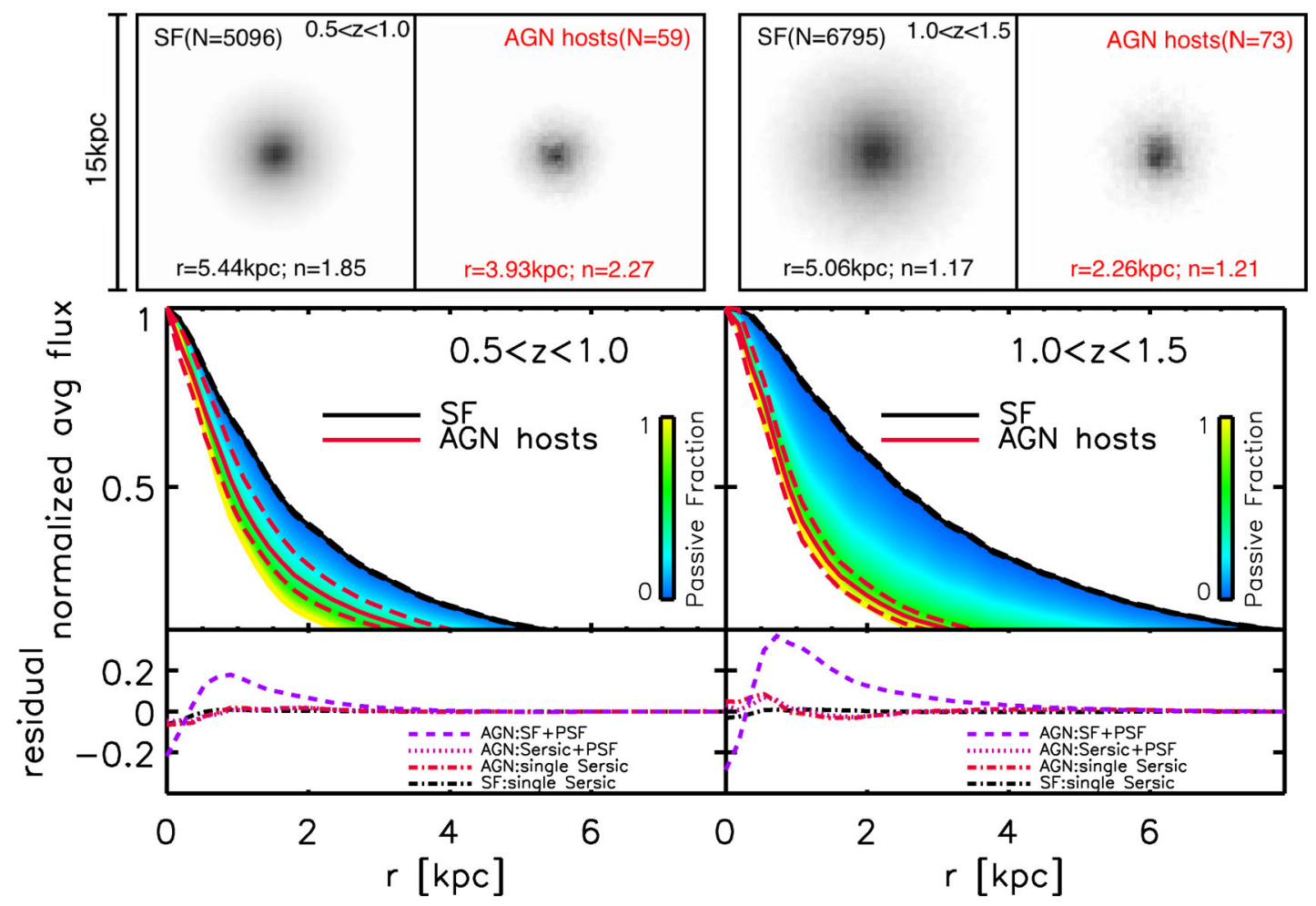

Figure 3. Top panels: Normalized stacked images of obscured AGN hosts and star-forming sources, displayed with a common grey-scale and selected as in Fig. 2, along with the half-light radius and the Sérsic index measured with GALFIT. Bottom panels: Average radial light profile obtained from the stacked images (red: obscured AGN hosts; black: star-forming sources). Solid and dashed lines represent the median profile and the $1 \sigma$ dispersion estimated with bootstrapping. The colored regions depict the radial profiles obtained after combining the stack of star-forming galaxies with the stack of $U V J$-selected red passive sources, with the contribution of the latter colour-coded as shown in the inset.Dash-dotted and dotted curves illustrate the residuals after fitting the stacks with a single Sérsic disc and a Sérsic+PSF two-component model, respectively. The blue dashed line shows the residuals after fitting the AGN stack with a PSF and a Sérsic disc fixed to that obtained for the population of star-forming sources.

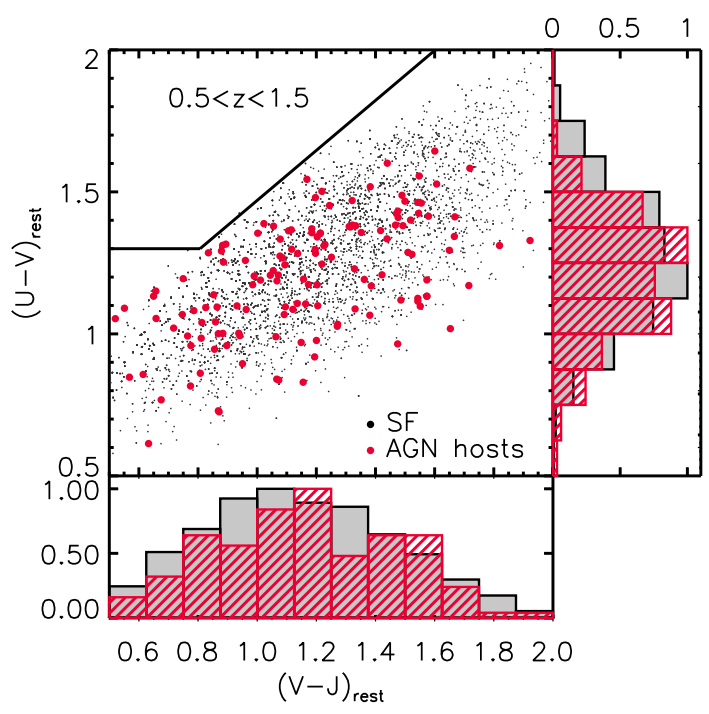

Figure 4. The UVJ rest-frame colours of AGN hosts (red dots, stellar component only) compared to the control sample of star-forming galaxies. The corresponding normalized histograms are shown along the $x$ - and $y$-axes. The hosts of obscured AGNs have optical/NIR colours typical of star-forming sources.

\section{DISCUSSION}

Obscured AGNs have negligible emission at UV/optical wavelengths, bringing the benefit of a minimal impact when characterizing the morphology of their underlying hosts with facilities like $H S T$. Here, we have shown that these hosts exhibit a radial light profile on average more compact than that of star-forming sources at similar mass, but not as steep as that observed in red passive objects (Fig. 3). At very first sight, this could be interpreted as the signature of 'transition galaxies', migrating from the population of disc-dominated blue sources to the red sequence of dead ellipticals, with the AGN possibly holding a negative feedback action responsible for the quenching of the star-forming activity. Numerical simulations, indeed, suggest that wet major mergers can trigger a burst of intense star formation accompanied by accretion of material on to a highly obscured black hole, eventually leading to the formation of a passive galaxy once the gas has been blown out (e.g. Hopkins et al. 2008). In this scenario though, we expect the obscured AGN to be observed only in the very first stages of the merger before the formation of the compact remnant and when the morphology is still highly perturbed. This is not consistent with the low fraction of mergers observed in our sample (Chang et al., in preparation), which also gives further support to the now widely accepted scheme in which the bulk of AGNs is not primarily linked to galaxy mergers. Besides, obscured AGN hosts have optical/NIR colours (Fig. 4) and sSFRs typical of star-forming galaxies (Chang et al., in preparation), which is hard to reconcile with sources in a quenching phase. 
More likely, we suggest that obscured AGNs are primarily hosted by star-forming galaxies having undergone a process of dynamical contraction, similar to the scenario recently proposed to explain the population of blue compact star-forming sources observed at $z \sim 1-2$. Cosmological hydrodynamical simulations of galaxy formation, indeed, suggest that highly perturbed gas-rich discs fed by cold streams at a high redshift can experience phases of dissipative contraction, leading to the formation of compact star-forming systems (Dekel \& Burkert 2014). In this scenario, the compaction is initially triggered by an episode of intense gas inflow involving counter-rotating streams or recycled gas as well as minor mergers. The inflow provides sufficient energy for maintaining a high level of turbulence and it is often associated with violent disc instabilities that drive gas to the central region of galaxies. It thus leads to a massive core with high gas fraction and enhanced star formation (Zolotov et al. 2015), and the inflow rate can also sustain gas accretion on to the central supermassive black hole, leading to the trigger of an AGN with moderately sub-Eddington luminosities (Bournaud et al. 2011). These disc instabilities can actually result in substantial central gas column densities with a peak distribution at $\log \left(N_{\mathrm{HI}}\right) \sim 10^{23} \mathrm{~cm}^{-2}$ (Bournaud et al. 2011). Gas compaction could thus lead to central extinctions with even larger values, consistent with the AGNs being highly obscured when they are hosted by compact star-forming sources.

Interestingly, it was found that compact star-forming sources at $z \sim 2$ seem more likely to host an X-ray bright AGN when compared to more extended sources (Barro et al. 2014), and that both clumpy and compact disc galaxies at $z \sim 2$ harbour a high AGN fraction (Trump et al. 2014). Here, we analysed a complete sample of AGNs with strong obscuration (defined by their X-ray-to-total IR AGN luminosity ratio) at $0.5 \leq z \leq 1.5$, showing that there could be an even stronger connection between the mechanisms driving obscured black hole accretion and compact galaxies, at least at these lower redshifts. Exploring further how this link is driven by the galaxy internal processes will require a larger sample of AGNs, which will be achieved, thanks to wider AGN surveys like those to be performed by $e$ ROSITA.

\section{ACKNOWLEDGEMENTS}

We thank the referee as well as A. Dekel and D. Elbaz for helpful comments and discussions. We acknowledge the contribution of the entire COSMOS collaboration as well as a financial support from ANR (\#ANR-12-JS05-0008-01).

\section{REFERENCES}

Barro G. et al., 2014, ApJ, 791, 52

Böhm A. et al., 2013, A\&A, 549, A46

Bournaud F., Dekel A., Teyssier R., Cacciato M., Daddi E., Juneau S., Shankar F., 2011, ApJ, 741, L33

Bruce V. A., Dunlop J. S., Mortlock A., Kocevski D. D., McGrath E. J., Rosario D. J., 2016, MNRAS, 458, 2391

Chabrier G., 2003, PASP, 115, 763

Cisternas M. et al., 2011, ApJ, 726, 57

Civano F. et al., 2016, ApJ, 819, 62

da Cunha E., Charlot S., Elbaz D., 2008, MNRAS, 388, 1595

da Cunha E. et al., 2015, ApJ, 806, 110

Dekel A., Burkert A., 2014, MNRAS, 438, 1870

Fiore F. et al., 2009, ApJ, 693, 447

Gabor J. M. et al., 2009, ApJ, 691, 705

Grogin N. A. et al., 2005, ApJ, 627, L97

Hopkins P. F., Hernquist L., Cox T. J., Kereš D., 2008, ApJS, 175, 356

Juneau S. et al., 2013, ApJ, 764, 176

Kocevski D. D. et al., 2012, ApJ, 744, 148

Kocevski D. D. et al., 2015, ApJ, 814, 104

Lacy M. et al., 2004, ApJS, 154, 166

Laigle C. et al., 2016, ApJS, 224, 24

Lanzuisi G. et al., 2013, MNRAS, 431, 978

Lanzuisi G. et al., 2015, A\&A, 578, A120

Le Floc'h E. et al., 2009, ApJ, 703, 222

Lilly S. J. et al., 2009, ApJS, 184, 218

Marchesi S. et al., 2016, ApJ, 817, 34

Merloni A. et al., 2014, MNRAS, 437, 3550

Mullaney J. R. et al., 2012, MNRAS, 419, 95

Peng C. Y., Ho L. C., Impey C. D., Rix H.-W., 2010, AJ, 139, 2097

Pierce C. M. et al., 2007, ApJ, 660, L19

Salvato M. et al., 2011, ApJ, 742, 61

Scoville N. et al., 2007, ApJS, 172, 1

Stern D., 2015, ApJ, 807, 129

Trump J. R. et al., 2014, ApJ, 793, 101

van der Wel A. et al., 2014, ApJ, 788, 28

Villforth C. et al., 2014, MNRAS, 439, 3342

Zolotov A. et al., 2015, MNRAS, 450, 2327

This paper has been typeset from a $\mathrm{T}_{\mathrm{E}} \mathrm{X} / \mathrm{L} \mathrm{T} \mathrm{E} \mathrm{X}$ file prepared by the author. 